\title{
Influência do Capital Social no Mercado de Crédito Rural
}

\author{
Roberto Arruda de Souza Lima ${ }^{1}$ \\ Ricardo Shirota ${ }^{2}$
}

Resumo: O trabalho analisa o efeito do capital social sobre o volume de negócios no mercado de crédito rural. No caso do crédito rural, o intermediário financeiro maximizador de lucro depara com uma importante restrição a este objetivo: historicamente, a taxa de juros nesse mercado tem sido fixada, pelo governo, abaixo do equilíbrio de mercado. Assim, o problema relevante para o intermediário financeiro é a minimização do seu custo. A hipótese, que foi verificada econometricamente (utilizando, para isso, um modelo de lógite), é que, ao contribuir para a redução dos custos transacionais, o nível de capital social afeta o volume de crédito rural. Assim, incentivos, em especial com apoio do setor público, para formação e manutenção de capital social permitiriam aumento da eficiência da intermediação financeira e, em conseqüência, maior desenvolvimento do setor rural.

Palavras-chaves: Capital social, crédito rural, lógite.

Classificação JEL: Z13, Q14, G14, L14, H81

\footnotetext{
${ }^{1}$ Doutorando em Economia Aplicada, Escola Superior de Agricultura "Luiz de Queiroz" (ESALQ/USP).

2 Professor doutor, Escola Superior de Agricultura “Luiz de Queiroz” (ESALQ/USP). rshirota@esalq.usp.br
} 
Abstract: This study analyses the effect of social capital on the volume of contracts in the rural credit market. It discusses how social capital contributes to the reduction of financial intermediation's transaction costs. A logit regression model was used to empirically test the effect of social capital on the volume of rural credit. The results indicate that the level of social capital affects the amount of rural credit. Thus, incentives to further increase and maintain social capital would increment the efficiency of financial intermediation and, as a consequence, help the rural sector's development.

Keywords: Social capital, rural credit, logit.

JEL Classification: Z13, Q14, G14, L14, H81

\section{Introdução}

O presente trabalho analisa o papel do capital social na oferta de crédito rural. A importância desta discussão está baseada tanto na pressuposição de que o mercado financeiro rural é relevante para o desenvolvimento da agricultura $^{3}$ (Almeida, 1994; Banco Mundial, 1989), quanto na hipótese - verificada neste estudo - de que o capital social pode influir na intermediação financeira, contribuindo para sua maior eficiência.

Um dos principais problemas na intermediação financeira é a assimetria de informações, uma relevante fonte de risco de crédito, sendo este definido como a possibilidade do devedor não pagar (Banco Mundial, 1989). Este problema repercute mesmo antes da formalização do contrato $^{4}$, de modo que o equilíbrio no mercado de financiamentos pode caracterizar-se por racionamento de crédito (Stiglitz \& Weiss, 1981).

No entanto, a assimetria de informação ocorre também após a formalização do contrato, seja em decorrência de determinadas ações tomadas pelo devedor e que não são observadas pelo credor, ou pelo resultado de

\footnotetext{
${ }^{3} \mathrm{O}$ desenvolvimento da agricultura é importante não apenas para os agentes diretamente ligados a este setor, mas para toda economia, ao assegurar a produção de alimentos e matérias-primas (a preços que não representem pressão inflacionária), além da geração de excedentes exportáveis.

${ }^{4}$ Havendo pouca informação, os credores selecionam os mutuários em termos mais amplos.
} 
ações da natureza (também observadas somente pelo devedor), e que afetam o retorno esperado do empreendimento. Nesta situação podem ocorrer problemas conhecidos como risco moral ${ }^{5}$.

Um sistema financeiro eficiente - com menores taxas de juros e, o que é particularmente importante no crédito rural, com maior volume de recursos - deve realizar suas transações a um custo mínimo. Isto depende do sucesso na avaliação das alternativas de investimentos e no direcionamento dos recursos disponíveis para estes, além do monitoramento dos tomadores. A intermediação financeira deve proporcionar o uso eficiente dos recursos (escassos) disponíveis (Shirota, 1996).

As características específicas do crédito rural favorecem o surgimento de economia de escala. Por exemplo, os custos ligados ao contato com o cliente nas propriedades rurais, em geral, distantes geograficamente da agência do credor. Os deslocamentos físicos (incluindo transporte, estadia, refeições, etc.), como nas visitas ao cliente (para contatos comerciais ou monitoramento), representam custos não desprezíveis que podem ser diluídos com o aumento do número de clientes em uma mesma região, que pudessem ser visitados em uma única viagem. Informações climáticas necessárias para acompanhar o risco de um cliente são válidas para clientes da mesma região. Enfim, características como a dispersão geográfica dos produtores rurais e a ocorrência de eventos generalizados na agricultura favorecem o surgimento de economia de escala no crédito rural.

Economia de escopo também está presente quando a indústria bancária opera com crédito rural. Economia de escopo ocorre sempre que o custo de produção conjunta é menor que o custo total de produção independente ${ }^{6}$ (Baumol et al., 1988). Assim, quando um banco já possui uma agência instalada em uma cidade operando com produtos diferentes do crédito rural (conta corrente, por exemplo), a introdução de operações de crédito rural poderá ser feita utilizando a estrutura física e pessoal já existente para diversas operações (como, por exemplo,

\footnotetext{
${ }^{5}$ A premissa básica de risco moral é que os indivíduos não têm o mesmo incentivo para cuidar da propriedade de outras pessoas com o mesmo zelo com que cuidam de seus próprios interesses, ou seja, há um incentivo menor em zelar pelo dinheiro emprestado do que pelos recursos próprios (Fry, 1995).

${ }^{6}$ Isto é, $C\left(y_{1}, y_{2}\right)<C\left(y_{1}, 0\right)+C\left(0, y_{2}\right)$.
} 
confecção de cadastro, processamento de dados, entre outras). Também poderá se utilizar de informações do cliente e das suas operações por fontes adicionais àquelas que teria se operasse exclusivamente com 0 crédito rural. Este é o caso, por exemplo, do cliente que movimenta a sua conta corrente ou realiza seus investimentos na mesma agência onde realiza financiamento rural (Berger et al., 1987). Esta situação permite o monitoramento do fluxo de caixa do tomador.

Os custos e as dificuldades na obtenção e processamento de informações tornaram o capital social um conceito com utilização crescente nas ferramentas de decisão referentes à oferta de crédito. Em sua concepção original, o capital social seria um facilitador da ação coletiva. No entanto, conforme destacado por Young (2001), este conceito foi ampliado, mostrando que o capital social afeta o comportamento dos indivíduos ao contribuir para a conscientização da extensão das relações interpessoais. O capital social permite que cidadãos resolvam problemas coletivos mais facilmente, pois as pessoas superam a inércia e o receio do free riding que normalmente impedem ações coletivas. Assim, o capital social reduz os custos de transações atrelados aos comportamentos oportunísticos. O menor incentivo a este tipo de comportamento reduz o risco moral, diminuindo os custos de monitoramento.

A informação e o capital social afetam o volume de negócios de forma substitutiva ${ }^{7}$. Coeteris paribus, quanto mais informação e/ou maior for o capital social, maior será o volume de negócios. Pode-se considerar que a soma de informações com capital social resulte em um vetor, que será denominado confiabilidade. Quanto mais alta for a confiança dos doadores de recursos na seleção, no monitoramento e na execução de seus créditos, maior volume de recursos será ofertado, aceitando menores taxas remuneratórias (menor será o risco). Assim:

$$
V C^{S}=f(j, c)
$$

\footnotetext{
${ }^{7}$ Ou seja, quando há pouca informação sobre determinado risco de crédito, um elevado capital social pode proporcionar uma maior segurança na concessão de um empréstimo. Por outro lado, se há grande quantidade (e qualidade) de informação disponível, não há necessidade de capital social para que o agente se sinta confortável para conceder um empréstimo.
} 
Sendo que:

$$
\frac{\partial V C^{S}}{\partial c}>0
$$

em que $V C^{S}=$ volume ofertado de crédito; $j=$ taxa de juros; e, $c=$ confiabilidade (informação + capital social). A Figura 1 representa esta relação hipotética.

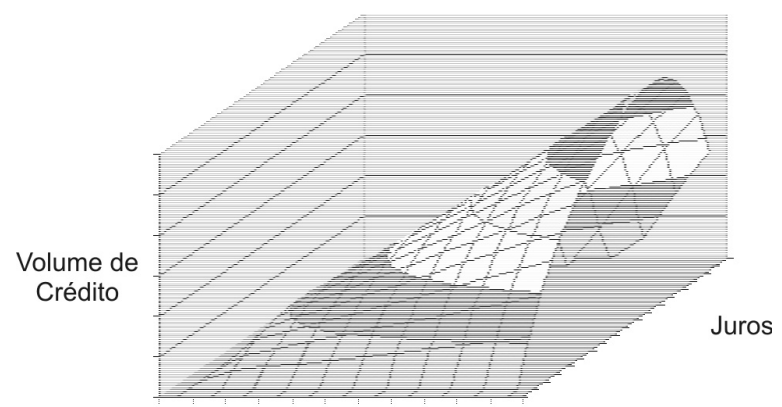

Figura 1 - Relação hipotética entre taxa de juros, confiabilidade e o volume de crédito ofertado.

A oferta de crédito pode ser representada conforme ilustrado na Figura 2.a. No Brasil, a taxa de crédito rural é fixada em nível inferior ao praticado em outras operações do mercado financeiro. Da mesma forma, o limite legal ${ }^{8}$ para operações entre empresas não financeiras (por exemplo, indústria de defensivos) e produtores rurais também é inferior às taxas praticadas em outras operações do mercado financeiro. Desta forma, podese considerar como relevante para as operações de crédito rural apenas o trecho de inclinação positiva da curva de oferta (Figura 2.b).

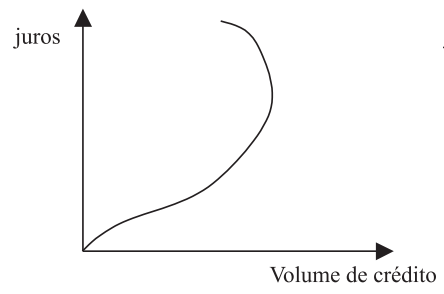

(a)

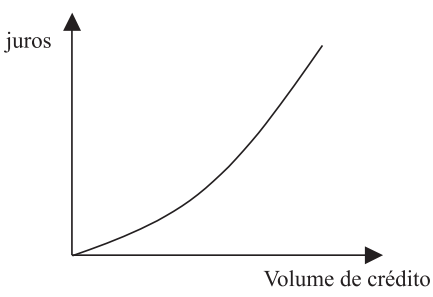

(b)

Figura 2 - Curva de oferta de crédito.

${ }^{8}$ Limite de $12 \%$ ao ano, estabelecido pelo Decreto $n^{\circ} 22.629$, de 07.04.33 — Lei da Usura. 
A curva de demanda por crédito, em uma situação de mercado livre, seria negativamente inclinada (Figura 3.a). No entanto, face à limitação dos juros em um patamar máximo, a curva de demandas apresentará um segmento infinitamente elástico (Figura 3.b), em que r* é o limite legal de juros.

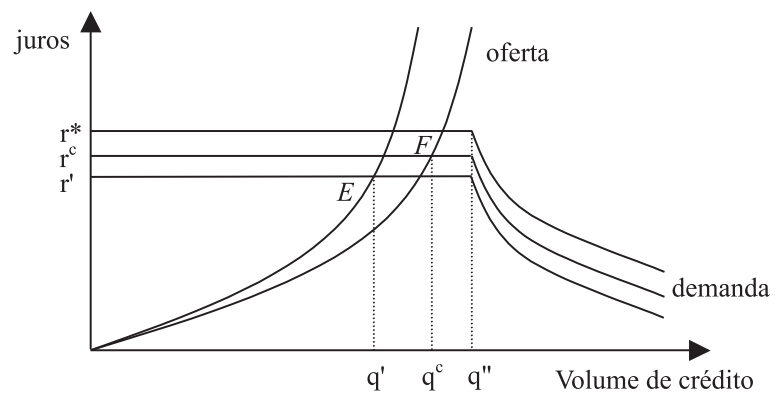

Figura 3 - Curva de demanda de crédito

Assim, o mercado de crédito rural pode ser representado conforme Figura 4. A presença de custos de transação implica que demandantes e ofertantes de crédito observarão taxas de juros diferentes. O tomador de crédito (demandante) fará sua escolha baseado na taxa de juros do financiamento ( $\left.\mathrm{r}^{*}\right)$. O credor (ofertante) fará sua escolha considerando a taxa de retorno do crédito ( $r$ '), isto é, a taxa de juros paga pelo tomador líquida dos custos de transação.

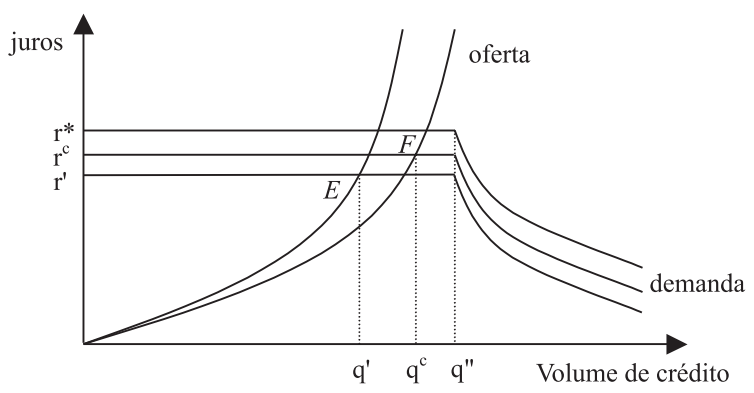

Figura 4 - Mercado de crédito rural com elevação da confiabilidade

Nesta situação, o equilíbrio de mercado ocorrerá no ponto $\boldsymbol{E}$, onde o volume de operações de crédito será q'. Note que a esta mesma taxa de juros, a demanda total é de q". Assim, há um racionamento de crédito no montante de (q" - q'). 
Uma elevação na confiabilidade (informação + capital social) reduziria o custo de transação. Isto deslocaria o equilíbrio de mercado até o ponto $\boldsymbol{F}$, resultando em uma elevação no volume de crédito (Figura 4), de q' para qc, reduzindo o racionamento.

\section{Metodologia}

Buscou-se verificar como o capital social afeta a probabilidade de um agricultor ter acesso ao crédito rural, ou seja, como o volume de negócios praticados no mercado é afetado. O modelo, para esta análise, pressupõe quatro hipóteses:

(a) o ambiente é exógeno. Não há variações institucionais (por exemplo, a eficiência do judiciário é a mesma em todas operações). Também não é considerada a ocorrência de fenômenos inesperados da natureza (como secas ou enchentes), que poderiam comprometer a oferta de crédito ao inviabilizar a produção agrícola;

(b) as informações complementares, como dados sobre clima e zoneamento agrícola, são de conhecimento dos ofertantes de crédito. $\mathrm{O}$ custo já foi incorrido e será o mesmo (ou seja, não há custo adicional) independente do nível de utilização destas informações;

(c) conforme o capital social se eleva, maior será a probabilidade de que ocorra uma transação de crédito; e,

(d) a relação entre a referida probabilidade e o capital social não é linear. Isto é, para baixo nível de capital social o acesso é pouco provável, assim como a partir de determinado nível de capital social, este deixaria de ser limitante ao acesso ao crédito.

Portanto, em vista das considerações teóricas apresentadas, postulase que a ocorrência de transação de crédito $(V)$ pode ser expressa pela seguinte função:

$$
V=f(G, K, W)
$$

em que $V=$ ocorrência de transação de crédito, assumindo valor 1 (ocorrido) ou 0 (não ocorrido); $G=$ Garantias oferecidas; $K=$ Capital social; e, $W=$ Outras variáveis. Este último termo buscará captar outras fontes que possam influenciar o volume de crédito, tais como a presença de 
agência bancária no município, facilidade de acesso, tamanho de contrato, entre outras. Em especial, são considerados os fatores que favorecem o surgimento de economias de escala e escopo no uso da informação.

O modelo de lógite será utilizado na verificação da relação entre a quantidade de operações de crédito rural e o capital social, conforme exposto a seguir'.

Admitindo que existem $k$ variáveis explanatórias para uma resposta quântica, o vetor-linha com os valores dessas variáveis explanatórias na j-ésima observação é:

$$
\mathbf{x}_{j}{ }^{\prime}=\left[\begin{array}{llll}
1 & x_{1 j} & \ldots & x_{k j}
\end{array}\right] \quad \operatorname{com} j=1, \ldots, n .
$$

em que $n$ é o número total de observações.

O correspondente vetor de parâmetros é:

$$
\boldsymbol{\beta}=\left[\begin{array}{c}
\beta_{0} \\
\beta_{1} \\
\vdots \\
\beta_{k}
\end{array}\right]
$$

No modelo de lógite admite-se que, dado $\boldsymbol{x}_{\mathrm{j}}$, a probabilidade de obter uma resposta favorável é:

$$
\mathrm{P}_{\mathrm{j}}=\left[1+\exp \left(-\mathbf{x}_{\mathrm{j}}{ }^{\prime} \beta\right)\right]^{-1}=\frac{1}{1+\exp \left(-\mathbf{x}_{\mathrm{j}}{ }^{\prime} \boldsymbol{\beta}\right)}
$$

Note-se que, se houver apenas uma variável explanatória, trata-se de uma curva logística com assíntota superior com ordenada 1.

Obtém-se:

$$
Q_{j}=1-P_{j}=\frac{\exp \left(-\mathbf{x}_{j}{ }^{\prime} \beta\right)}{1+\exp \left(-\mathbf{x}_{j}{ }^{\prime} \beta\right)}
$$

\footnotetext{
${ }^{9}$ A descrição do modelo é a apresentada por Hoffmann (2000).
} 


$$
\frac{P_{j}}{Q_{j}}=\exp \left(\mathbf{x}_{j}{ }^{\prime} \beta\right)
$$

Então:

$$
Y_{j}=\ln \frac{P_{j}}{Q_{j}}=\mathbf{x}_{j}{ }^{\prime} \boldsymbol{\beta}=\beta_{0}+\beta_{1} x_{1 j}+\cdots+\beta_{k} x_{k j}
$$

que é o lógite correspondente a $P_{j}$. Note-se que, partindo do modelo não linear [eq. (6)], o lógite é, por construção, uma função linear nas variáveis explanatórias. Quando $P_{j}$ varia de zero a 1, o lógite varia de $-\infty$ a $+\infty$. Os parâmetros do modelo de lógite são estimados pelo método da máxima verossimilhança.

O modelo proposto [eq. (3)] é estimado utilizando o lógite representado pela eq. (9). A análise foi restrita a apenas um estado - São Paulo - para evitar a interferência de outras variáveis regionais, como, por exemplo, a diferente eficiência do Judiciário em cada estado. Assim, o índice $j$ da eq. (9) representa cada um dos municípios do Estado de São Paulo.

O percentual de Unidades de Produção Agropecuária (UPA ${ }^{10}$ que receberam crédito, em cada um dos municípios do Estado de São Paulo, serviu como proxy do crédito transacionado [variável $V$ da eq. (3)]. Na eq. (9), $Y_{j}$ é o valor deste percentual, para cada município $j$, obtido do LUPA ${ }^{11}$.

$\mathrm{O}$ vetor $\mathbf{x}_{j}$, apresentado na eq. (9), é composto por sete variáveis explanatórias (LPROD, CAPSOC, ICMS, OPCRED, AGBCO, AREA e CRIMES) para cada município $j$.

A capacidade do tomador oferecer garantias afeta a probabilidade de obter crédito. É pressuposto que quanto maior for a produção, maior deverá ser o patrimônio do produtor e, consequentemente, as alternativas de garantias que poderão ser oferecidas ao credor. Assim, a variável LPROD - correspondendo ao valor da receita (na forma logarítmica) de produção animal e vegetal, por município, conforme dados do Instituto

\footnotetext{
${ }^{10}$ Unidade de Produção Agropecuária (UPA) é o imóvel rural (não considerados os imóveis utilizados somente para lazer), entendido como o conjunto de propriedades contíguas do(s) mesmo(s) proprietário(s) (São Paulo, 1999).

${ }^{11}$ Levantamento das Unidades de Produção Agropecuária (LUPA), referente ao Estado de São Paulo, safra de 1995/96, elaborado pela Coordenadoria de Assistência Técnica Integral - CATI.
} 
Brasileiro de Geografia e Estatística (IBGE, 1997) - foi adotada como proxy da variável $G$ da eq. (3).

O percentual de UPAs que fazem parte de cooperativas, associações ou sindicatos de produtores foi utilizado como medida da quantidade de capital social [variável $K$ da eq. (3)], em um determinado município. Buscando reduzir o risco de dupla contagem, para cada município, a variável utilizada foi o valor máximo entre estas três participações. Assim, a variável CAPSOC serviu como proxy do capital social.

Outras variáveis foram introduzidas no modelo para captar o interesse da instituição financeira operar no município, como reflexo de diferenças nas condições de mercado e de economias de escala e de escopo. Neste sentido, foram incluídas as variáveis referentes ao porte financeiro do município (variável ICMS, correspondendo ao percentual de participação dos municípios na arrecadação do ICMS) e ao volume de operações de crédito (variável OPCRED). O número de agências bancárias por mil habitantes (variável AGBCO) foi introduzido no modelo para captar a concorrência e a atratividade do mercado financeiro local (não apenas de crédito rural). Também foram consideradas as áreas utilizadas pelas UPAs (variável AREA), por município, assumindo que o custo de monitoramento é uma função da área a ser estudada.

A concretização da produção agropecuária não é o bastante para o credor. É necessário que ela esteja disponível (para garantia ou comercialização) e que parcela de sua receita seja direcionada para a efetiva liquidação do contrato. Para captar a segurança do credor quanto a este tópico, foi incluída uma variável explanatória (CRIMES) que indica a proporção de ocorrências de crimes contra o patrimônio, verificada no município, para cada habitante ${ }^{12}$.

O Estado de São Paulo, atualmente, é composto por 645 municípios. Destes, dezoito foram criados entre os anos de 1993 e 1996, tendo sido efetivamente instalados em 01 de janeiro de 1997. Como os dados utilizados referem-se à safra 95/96, as informações de cada um destes novos municípios foram agregadas aos dados dos municípios originais (anteriores ao desmembramento). Os municípios que não constavam do

${ }^{12}$ Informações obtidas junto ao banco de dados Informações dos Municípios Paulistas, da Fundação Sistema Estadual de Análise de Dados - SEADE. 
Censo Agropecuário ou do LUPA foram desconsiderados neste estudo. Os municípios que não apresentam estatísticas completas (dados não disponíveis ou classificados como sigilosos) na base de dados do SEADE foram excluídos do presente estudo ${ }^{13}$. As estatísticas apresentadas no LUPA referentes ao município de Cabrália Paulista estão inconsistentes ${ }^{14}$. Assim, este município foi excluído do presente estudo. A participação da agropecuária na cidade de São Paulo (Capital) é notoriamente marginal. No entanto, a expressão desta cidade nas variáveis quantitativas referentes ao ICMS ${ }^{15}$ e movimentação financeira é muito significativa. Para que não houvesse a influência desta capital nos resultados, não relevante ao tema analisado, este município também foi retirado da amostra.

\section{Resultados e discussão}

As três medidas utilizadas na estimativa do capital social apresentaram estatísticas semelhantes com relação à média aritmética e às medidas de dispersão (Tabela 1) embora o coeficiente de correlação entre elas seja baixo (Tabela 2), indicando que apresentam comportamentos distintos. As participações dos produtores rurais dos municípios do Estado de São Paulo em organizações sociais exibem grande amplitude, variando de $0 \%$ (isto é, nenhum proprietário participando em cooperativas, associações e sindicatos) até valores próximos de $100 \%$ de participação.

\footnotetext{
${ }^{13}$ Trata-se de uma redução de $22,22 \%$ na amostra. Isto pode potencialmente introduzir um viés na amostra. Como atenuante a este problema, destaca-se que os municípios eliminados não estão concentrados geograficamente e apresentam média e desvio padrão $(0,1504$ e 0,1331 , respectivamente), referentes ao percentual de UPAs que receberam crédito rural, próximos aos da amostra analisada (0,1502 e 0,1419, respectivamente).

${ }^{14}$ Foi indicada a presença de 275 UPAs que fazem parte de cooperativas, sendo que o município de Cabrália Paulista possuía, na época, apenas 163 UPAs.

${ }^{15}$ O percentual de participação da capital na arrecadação de ICMS é de 27,17\%, sendo que a segunda maior cidade possui índice de $2,93 \%$ e a média da amostra é de 0,10\% .
} 
Tabela 1. Estado de São Paulo: média aritmética e medidas de dispersão das participações percentuais em cooperativas, associações e sindicatos e variável

CAPSOC, por município, safra 1995/96.

\begin{tabular}{lcccc}
\hline \multicolumn{1}{c}{ Estatística } & $\begin{array}{c}\text { Participação em } \\
\text { Cooperativas }\end{array}$ & $\begin{array}{c}\text { Participação em } \\
\text { Associações }\end{array}$ & $\begin{array}{c}\text { Participação em } \\
\text { Sindicatos }\end{array}$ & CAPSOC \\
\hline Média & 0,3866 & 0,2139 & 0,3092 & 0,4551 \\
Desvio padrão & 0,2417 & 0,1972 & 0,2030 & 0,2162 \\
Mínimo & 0,0000 & 0,0000 & 0,0000 & 0,0000 \\
Máximo & 0,9706 & 0,9567 & 1,0000 & 1,0000 \\
Amplitude & 0,9706 & 0,9567 & 1,0000 & 1,0000 \\
\hline
\end{tabular}

Fonte: Resultado da pesquisa

Tabela 2. Estado de São Paulo: coeficientes de correlação entre as participações percentuais em cooperativas, associações e sindicatos, por município, safra 1995/96.

\begin{tabular}{cccc}
\hline & Cooperativas & Associações & Sindicatos \\
\hline Cooperativas & 1 & & \\
Associações & 0,540356 & 1 & 1 \\
Sindicatos & 0,381718 & 0,461414 & 1 \\
\hline
\end{tabular}

Fonte: Resultado da pesquisa

A Tabela 3 apresenta estatísticas referentes às variáveis explanatórias utilizadas no modelo.

Tabela 3. Média aritmética e medidas de dispersão das variáveis CRIMES, em 1997, AGBCO, OPCRED, LPROD, ICMS e AREA, safra 1995/96, por município, Estado de São Paulo.

\begin{tabular}{lcccccc}
\hline \multicolumn{1}{c}{ Estatística } & AGBCO & OPCRED & LPROD & CRIMES & ICMS & AREA \\
\hline Média & 0,2288 & 15,4021 & 9,0905 & 0,0132 & 0,1035 & 31,4484 \\
Desvio padrão & 0,1038 & 2,1537 & 1,3325 & 0,0080 & 0,2502 & 26,1165 \\
Mínimo & 0,0173 & 10,6170 & 1,3863 & 0,0014 & 0,0056 & 0,0250 \\
Máximo & 0,8097 & 21,8624 & 11,9236 & 0,0601 & 2,9282 & 141,8590 \\
Amplitude & 0,7924 & 11,2455 & 10,5373 & 0,0587 & 2,9227 & 141,8340 \\
\hline
\end{tabular}

Fonte: Resultado da pesquisa

O teste da razão de verossimilhança foi utilizado para verificar se o modelo proposto [eq. (3)], estimado utilizando o lógite representado pela eq. (9) e as informações referentes às variáveis descritas na seção 2 deste artigo, é melhor que um modelo em que todos coeficientes fossem nulos (ou seja, é um teste global de significância). A razão de verossimilhança obtida foi igual a 8.605,5656 com sete graus de liberdade, que é altamente significativa, $\operatorname{com} p<0,0001$. Portanto, ao menos um dos coeficientes não é nulo. Os testes individuais dos coeficientes foram todos significativos com $p<0,01$ (Tabela 4). 
Tabela 4. Análise dos estimadores de máxima verossimilhança.

\begin{tabular}{cccccc}
\hline & GL & Estimador & Desvio padrão & Wald Qui-quadrado & Valor- $p$ \\
\hline Intercepto & 1 & $-7,74310$ & 0,088600 & $7.641,6104$ & $<0,0001$ \\
LPROD & 1 & 0,48800 & 0,009480 & $2.648,4984$ & $<0,0001$ \\
CAPSOC & 1 & 1,23610 & 0,030900 & $1.596,1674$ & $<0,0001$ \\
ICMS & 1 & $-1,05200$ & 0,046900 & 503,4251 & $<0,0001$ \\
OPCRED & 1 & 0,04080 & 0,004570 & 79,8163 & $<0,0001$ \\
AGBCO & 1 & 1,89640 & 0,073800 & 660,3616 & $<0,0001$ \\
AREA & 1 & $-0,00618$ & 0,000254 & 593,1716 & $<0,0001$ \\
CRIMES & 1 & $-2,80020$ & 1,017000 & 7,5812 & 0,0059 \\
\hline
\end{tabular}

Fonte: Resultado da pesquisa

De acordo com a estimativa assim obtida dos parâmetros, o modelo de lógite [eq. (9)] é:

$$
\begin{aligned}
& Y=-7,7431+0,4880 \text { LPROD }+1,2361 \text { CAPSOC }-1,052 \text { ICMS }+ \text { (10) } \\
& + \text { 0,0408 OPCRED + 1,8964 AGBCO - 0,00618 AREA - 2,8002 CRIMES }
\end{aligned}
$$

Os coeficientes apresentaram os sinais esperados, com exceção do relativo à variável ICMS. Esta variável foi introduzida no modelo para captar o interesse de instituições financeiras operarem nos municípios. No entanto, a participação do setor primário na economia varia de município para município. Assim, a influência dos municípios industrializados que, em geral, apresentam elevados valores referentes à variável ICMS e baixa vocação rural (por definição), ocorre no sentido inverso do esperado. Ou seja, valores elevados da variável ICMS indicam forte industrialização nestes municípios e valores baixos no lógite, o que teria implicado no sinal negativo do coeficiente relativo à variável ICMS.

As razões de chance (odds ratio) obtidas estão apresentadas na Tabela 5. Os valores mais elevados das variáveis LPROD, CAPSOC e AGBCO implicam em maior probabilidade de ocorrência de crédito rural, enquanto um aumento da criminalidade (elevação do valor da variável CRIMES) e no valor da variável ICMS reduz esta probabilidade. Variações nas variáveis OPCRED e AREA afetam muito pouco a probabilidade de ocorrência de crédito rural. 
Tabela 5. Estimativas de razão de chances

\begin{tabular}{cccc}
\hline & & \multicolumn{2}{c}{ Intervalo de confiança (limites) } \\
\cline { 3 - 4 } Efeito & Estimativa & Inferior & Superior \\
\hline LPROD & 1,629 & 1,599 & 1,660 \\
CAPSOC & 3,442 & 3,240 & 3,658 \\
ICMS & 0,349 & 0,319 & 0,383 \\
OPCRED & 1,042 & 1,032 & 1,051 \\
AGBCO & 6,662 & 5,765 & 7,698 \\
AREA & 0,994 & 0,993 & 0,994 \\
CRIMES & 0,061 & 0,008 & 0,446 \\
\hline
\end{tabular}

Fonte: Resultado da pesquisa

Para avaliar a qualidade de ajuste (goodness of fit) do modelo utilizamse medidas que indicam a acurácia com a qual o modelo se aproxima dos dados observados (assim como o $\mathrm{R}^{2}$ no modelo de regressão linear). Quando a variável dependente é qualitativa, a acurácia pode ser avaliada em termos do ajuste entre as probabilidades calculadas e as freqüências das respostas observadas (Maddala, 1990). Foram obtidas as seguintes associações entre as respostas observadas e as probabilidades estimadas: (i) percentual concordante $=64,2$; (ii) percentual discordante $=34,7$; e, (iii) percentual de empates $=1,1$. A partir destas informações, foram geradas quatro estatísticas que avaliam a qualidade do modelo: (i) Somers' $\mathrm{D}=0,295$; (ii) Gamma =0,298; (iii) Tau-a $=0,075$; e, (iv) $\mathrm{c}=0,648$. A estatística Gamma tem a interpretação de redução proporcional no erro (DeMaris, 1992), assim como a estatística Somers’ D. Os resultados obtidos sugerem que são realizados cerca de $30 \%$ menos prognósticos incorretos em relação sobre qual município terá maior proporção de operações de crédito rural quando são utilizadas as informações de acordo com o modelo. A estatística Tau-a, para a amostra analisada, poderia apresentar um valor máximo de 0,254 (caso $100 \%$ dos pares fossem concordantes). Assim, o valor obtido $(0,075)$ representa $29,5 \%$ do valor máximo, corroborando com os resultados obtidos nas estatísticas Somers' D e Gamma. Como o percentual de empates foi relativamente baixo, o valor da estatística c aproxima-se do percentual de pares concordantes. As quatro estatísticas indicam que a qualidade de ajuste do modelo é boa.

No entanto, a medida da qualidade do ajuste a partir das associações entre as respostas observadas e as probabilidades estimadas apresenta deficiências. Em particular, não há um padrão na literatura para confrontar as medidas obtidas do percentual de prognósticos corretos. Adicionalmente, um 
prognóstico incorreto quando a probabilidade estimada for, por exemplo, $51 \%$, pode ser considerado um erro "menor" que o prognóstico incorreto com probabilidade estimada de $99 \%$. Ainda assim, estas medidas são indicadores adequados da qualidade do modelo ${ }^{16}$ (Aldrich \& Nelson, 1984).

O número de UPAs que receberam crédito rural, em percentagem, é estimado pelo modelo substituindo os valores obtidos através da eq. (10) na eq. (6). Os valores estimados apresentaram uma distribuição geográfica similar aos dados reais (Figura 5). A Tabela 6 apresenta estatísticas referentes a estes percentuais (estimado e real).

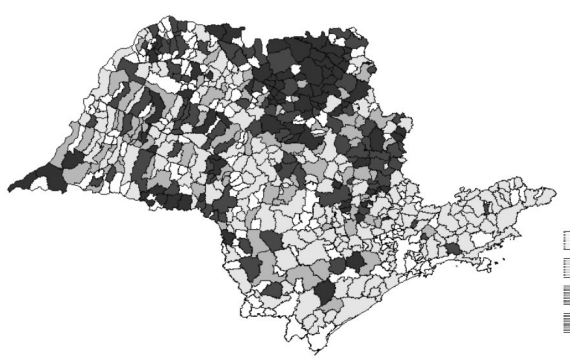

(a) Efetivo

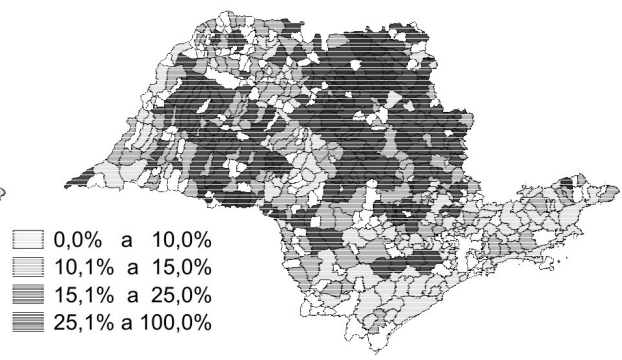

(b) Estimativa

Figura 5 - Estado de São Paulo: distribuição geográfica dos municípios (a) de acordo com a percentagem de UPAs que efetivamente receberam crédito rural e (b) de acordo com a percentagem de UPAs que teriam recebido crédito rural de acordo com o modelo, safra 1995/96

Fonte: Resultado da pesquisa

Tabela 6. Estado de São Paulo: média aritmética e medidas de dispersão dos percentuais de UPAs que receberam crédito rural, números reais e estimativas, por município, safra 1995/96

\begin{tabular}{lcc}
\hline \multicolumn{1}{c}{ Estatística } & Real & Estimado \\
\hline Média & 0,1479 & 0,1387 \\
Desvio padrão & 0,1394 & 0,0723 \\
Mínimo & 0,0000 & 0,0009 \\
Máximo & 0,8655 & 0,3789 \\
Amplitude & 0,8655 & 0,3780 \\
\hline
\end{tabular}

Fonte: Resultado da pesquisa

${ }^{16}$ Pode-se avaliar a qualidade do modelo através do número de prognósticos incorretos. A principal desvantagem neste caso surge quando há eventos que ocorrem com altas ou baixas probabilidades, situações em que a maioria dos modelos será considerada adequada por este critério (Ameniya, 1981). 
Destaque-se que características particulares de regiões como a de Campinas e a de Ituverava não foram adequadamente captadas pelo modelo. A presença de agroindústrias que exercem forte influência sobre as UPAs, como usinas e esmagadoras de grãos, assegurando a comercialização agrícola (e, consequentemente, o fluxo de caixa do empreendimento rural) caracteriza estas regiões e pode influenciar o acesso ao crédito. No entanto, nenhuma das variáveis presentes no modelo capta a presença ou a influência de agroindústrias nos municípios analisados.

\section{Conclusões}

A utilização do conceito de capital social tem sido crescente como alternativa de redução de custos de transação diante de informação imperfeita. O capital social reduz os custos de transações atrelados aos comportamentos oportunísticos, diminuindo, assim, os custos de seleção, monitoramento e de execução de contratos.

Uma importante constatação é que o capital social favorece o desenvolvimento da agricultura na medida em que torna o mercado de crédito rural mais eficiente e aumenta o volume de financiamentos. Quanto mais alta for a confiança (somatório de informação e capital social) dos ofertantes de recursos na seleção, no monitoramento e na execução de seus créditos, tanto maior será o volume de recursos ofertado, com menores taxas de juros (menor será o risco).

Os resultados empíricos obtidos no presente estudo mostram que, de fato, o volume de crédito rural contratado no Estado de São Paulo é afetado pela variável capital social. Esse resultado tem importantes implicações de natureza política. Por exemplo, a eficiência no mercado de crédito rural poderia ser obtida via redução do risco de crédito, aperfeiçoando e tornando mais disponíveis as informações sobre os mutuários. Isto porque, dentre os custos de transação, aqueles ligados à informação são relevantes e constituem, muitas vezes, barreiras à entrada de novos financiadores e à expansão desse mercado. Neste sentido, instituições locais, tais como aquelas ligadas ao microcrédito e agências locais de crédito, e a estrutura do Banco do Brasil podem exercer papel decisivo para tornarem disponíveis mais e melhores informações para o mercado, 
desde que adequadamente incentivadas (caso contrário, haverá subinvestimento na geração de informações ou restrição à disponibilidade daquelas que forem geradas).

Desta forma, o mercado de crédito rural pode desenvolver-se mais, com incremento no volume de negócios, através de incentivos que favoreçam não apenas a geração e a disponibilidade de informação, mas também a criação e manutenção de capital social. Iniciativas sociais, como as associações horizontais (cooperativas, associações e sindicados de produtores rurais) poderiam ser apoiadas pelo governo como forma de redução de custos de transação e fortalecimento do mercado financeiro. Isto para que ocorra um aumento da eficiência da intermediação financeira e, em conseqüência, sejam elevados os volumes de recursos ofertados para a agricultura. As características de não-rivalidade e nãoexclusividade do capital social implicam em subinvestimento privado na sua formação e manutenção. Isto ressalta a importância do apoio do setor público na construção de capital social.

\section{Referências bibliográficas}

ALDRICH, J.H.; NELSON, F.D. Linear probability, logit and probit models. Newbury Park: Sage Publications, 1984. 95p. (SAGE university paper series, 45)

ALMEIDA, A. Mercados informais como fonte alternativa de liquidez para os agricultores. Piracicaba, 1994. 204p. Dissertação (M.S.) - Escola Superior de Agricultura “Luiz de Queiroz”, Universidade de São Paulo. AMENIYA, T. Qualitative response models: a survey. Journal of Economic Literature, v.19, n.4, p.1483-1536, Dec. 1981.

BANCO MUNDIAL. Relatório sobre o desenvolvimento mundial: sistemas financeiros e desenvolvimento. Rio de Janeiro: Fundação Getúlio Vargas, 1989. 265p.

BAUMOL, W.T.; PANZAR, J.C.; WILLIG, R.D. Contestable markets and theory of industry structure. New York: Hardcourt Brace Javanovich, 1988. 538p.

BERGER, A.N.; HANWECK, G.A.; HUMPHREY, D.B. Competitive via- 
bility in banking: scale, scope, and product mix economies. Journal of Monetary Economics, v.20, p.501-520, 1987.

DeMARIS, A. Logit modeling: practical applications. Newbury Park: Sage Publications, 1992. 87p. (SAGE university paper series, 86)

FRY, M.J. Money, interest, and banking in economic development. Baltimore: The Johns Hopkins University Press, 1995. 568p.

HOFFMANN, R. Variável dependente binária: lógite e próbite. Piracicaba: ESALQ, Depto. Economia, Administração e Sociologia, 2000. 23p. (Série Didática, 126)

INSTITUTO BRASILEIRO DE GEOGRAFIA E ESTATÍSTICA. Censo agropecuário 1995-96 (compact disc). Rio de Janeiro, 1997. n.19

MADDALA, G.S. Limited-dependent and qualitative variables in econometrics. Cambridge: Cambridge University Press, 1990. 401p.

SÃO PAULO (Estado). Coordenadoria de Assistência Técnica Integral. Levantamento das unidades de produção agropecuária: estatísticas agrícolas, Estado de São Paulo 1995/96. São Paulo: CATI, 1997. http:// www.cati.sp.gov.br/servicos/lupa/ (03 out. 2002)

SHIROTA, R. Efficiency in financial intermediation: a study of the Chilean banking industry. Columbus, 1996. 138p. Thesis (Ph.D.) - The Ohio State University.

STIGLITZ, J.E.; WEISS, A. Credit rationing in markets with imperfect information. American Economic Review, v.71, n.3, 393-411, June 1981.

YOUNG, F.W. Review essay: Putnam's challenge to community sociology, Rural Sociology, v.66, n.3, p.468-474, Sep. 2001. 